\title{
Control of Femoral Cannulation with a ProGlide Pre-Closure Device during Cardiac Surgery: Is It Reliable?
}

\author{
Chang Hun Kim, M.D., Min Ho Ju, M.D., Mi Hee Lim, M.D., Chee-Hoon Lee, M.D., Hyung Gon Je, M.D., Ph.D. \\ Department of Thoracic and Cardiovascular Surgery, Pusan National University Yangsan Hospital, Medical Research Institute, Pusan National University School of \\ Medicine, Yangsan, Korea
}

\section{ARTICLE INFO}

Received January 19, 2021

Revised March 13, 2021

Accepted March 17, 2021

Corresponding author

Min Ho Ju

Tel $82-55-360-2127$

Fax 82-55-360-2157

E-mail deicidepan@naver.com

ORCID

https://orcid.org/0000-0001-7839-8598
Background: The use of ProGlide as a percutaneous vascular closure device in cardiac surgery remains inconclusive. This study investigated the clinical outcomes of using ProGlide in the percutaneous cannulation of femoral vessels in adult cardiac surgery.

Methods: From September 2017 to July 2018, 131 consecutive patients underwent femoral vessel cannulation during cardiac surgery. The ProGlide (Abbott Vascular Inc., Santa Clara, CA, USA) with percutaneous cannulation was used in 118 patients (mean age, $55.7 \pm 15.5$ years). The accessibility of femoral cannulation was evaluated through preoperative computed tomography. For cannulation, sonography was routinely used. The postoperative ankle-brachial index (ABI) was used to evaluate femoral artery stenosis.

Results: Of the 118 patients, 112 (94.9\%) and 6 (5.1\%) underwent minimally invasive cardiac surgery and median sternotomy, respectively. Most femoral cannulations were performed on the right side (98.3\%) using 15F to 19F arterial cannulas. The technical success rate of cannulation with ProGlide was $99.2 \%$, with no delayed bleeding or cannulation site-related complications during hospitalization. During follow-up, only 1 patient showed femoral artery stenosis with claudication and was treated with interventional balloon angioplasty. The postoperative ABI revealed no significant difference in functional stenosis between the cannulation and non-cannulation sides ( $n=86$; cannulation vs. non-cannulation, $1.2 \pm 0.1$ vs. $1.1 \pm 0$ ).

Conclusion: Percutaneous femoral cannulation with ProGlide was safe and feasible in adult cardiac surgery. This technique may be a good alternative option in patients requiring femoral vessel cannulation for cardiac surgery.

Keywords: Femoral cannulation, Femoral artery, ProGlide, Cardiac surgery

\section{Introduction}

The femoral artery (FA) is the most commonly used blood vessel when peripheral arterial cannulation is required for cardiopulmonary bypass (CPB). In recent years, use of the FA has been increasing gradually with the widespread application of redo cardiac surgery or minimally invasive cardiac surgery (MICS) [1]. Traditionally, when FA cannulation is performed, a small groin incision is made to expose the FA [2].

More recently, however, various types of percutaneous vascular closure devices (PVCDs) have been developed and used for cannulation of the FA [3]. In fact, PVCDs were developed for hemostasis after an interventional procedure.
The use of PVCDs has been increasing concomitantly with the expansion of interventions after advances in technology and the accumulation of experience in clinical practice. Previous reports have confirmed the safety and effectiveness of PVCDs [3-6]. As clinicians have gained experience with using PVCDs, their use has expanded, including in the field of cardiac surgery [7].

However, cardiac surgery and interventional procedures have many differences, including cannula size, procedural time, heparin concentration, and systemic hypothermia. Despite these differences, the literature on the use of PVCDs in cardiac surgery is insufficient. In addition, only a few studies have evaluated FA stenosis after PVCD use, especially in cardiac surgery. 
Since September 2017, we have been routinely using the totally percutaneous method with ProGlide (Abbott Vascular Inc., Santa Clara, CA, USA) during femoral cannulation. Thus, this study investigated the clinical outcomes of this method and assessed the feasibility and safety of PVCD use in cardiac surgery.

\section{Methods}

\section{Study population}

This observational, retrospective study included patients who underwent cardiac surgery at a single center between September 2017 and July 2018. All data were collected from the institutional surgical database. During the study period, a total of 131 adult ( $>17$ years old) patients underwent peripheral cannulation using the FA for $\mathrm{CPB}$. All patients underwent preoperative computed tomography angiography (CTA) to assess the suitability of peripheral cannulation, focusing on the size, degree of calcification, tortuosity, and location of bifurcation. Abnormalities (atherosclerosis, deformity, and variation) of the thoracic and abdominal great vessels were also assessed. ProGlide was used in 118 patients for FA cannulation. Patients who underwent an open surgical technique $(n=13)$ were excluded from this study because the use of ProGlide was inappropriate based on their CTA findings (FA less than $5 \mathrm{~mm}$ in diameter, or

Table 1. Baseline characteristics $(n=18)$

\begin{tabular}{lc}
\hline \multicolumn{1}{c}{ Characteristic } & Value \\
\hline Age $(\mathrm{yr})$ & $55.7 \pm 15.5$ \\
Male & $56(47.5)$ \\
Height $(\mathrm{cm})$ & $161.0(142.5-186.9)$ \\
Weight $(\mathrm{kg})$ & $60.8(40.5-87.6)$ \\
Body mass index $\left(\mathrm{kg} / \mathrm{m}^{2}\right)$ & $23.5 \pm 3.5$ \\
Body surface area $\left(\mathrm{m}^{2}\right)$ & $1.7 \pm 0.2$ \\
Hypertension & $50(42.4)$ \\
Diabetes mellitus & $13(11.0)$ \\
NYHA functional class III or IV & $43(36.4)$ \\
Preoperative hemodialysis & $2(1.7)$ \\
Left ventricular ejection fraction & $61.4 \pm 10.5$ \\
Euroscore II & $4.0 \pm 7.2$ \\
Size of FA on preoperative CTA & \\
Smaller than 7 mm & $21(17.8)$ \\
$7<$ size $\leq 8$ mm & $26(22.0)$ \\
$8<$ size $\leq 9$ mm & $20(16.9)$ \\
Larger than 9 mm & $51(43.2)$ \\
\hline
\end{tabular}

Values are presented as mean \pm standard deviation, number $(\%)$, or median (range).

NYHA, New York Heart Association; FA, femoral artery; CTA, computed tomography angiography. severely calcified FA). The cause of FA cannulation was MICS in 112 patients (94.9\%) and redo cardiac surgery in 6 patients (5.1\%). Details of the patients' characteristics are described in Table 1.

The study was approved by the institutional review board of Pusan National University Yangsan Hospital, and the requirement for individual consent was waived (IRB approval no., 04-2019-005).

\section{Use of ProGlide with the pre-marking method}

After the anesthetic procedure, the patient's legs were slightly flexed and externally rotated, as in the saphenous vein harvesting position. We believe that puncturing the common FA just above the bifurcation is the most important factor for increasing the success rate of cannulation and preventing stenosis or occlusive complications with ProGlide use. For this, we checked the path of the FA under sonographic guidance and pre-marked the path on the skin with a permanent marker (Fig. 1). In addition, we checked the bifurcation of the common FA by sonography and performed cross-marking on the skin of the bifurcation site to determine the appropriate puncture site. We also confirmed the location of the femoral vein (FV).

At the beginning of surgery, the FV and FA were punctured percutaneously, a 5F sheath was inserted, and the patency of the sheath was checked with a saline injection. We then made the main incision for cardiac surgery. After careful hemostasis of the main wound, systemic heparinization was performed for cannulation. First, percutaneous venous cannulation was performed under confirmation of the guidewire by transesophageal echocardiography (TEE) via a pre-inserted $5 \mathrm{~F}$ sheath. Subsequently, we connected a venous cannula to the $\mathrm{CPB}$ circuit and started arterial can-

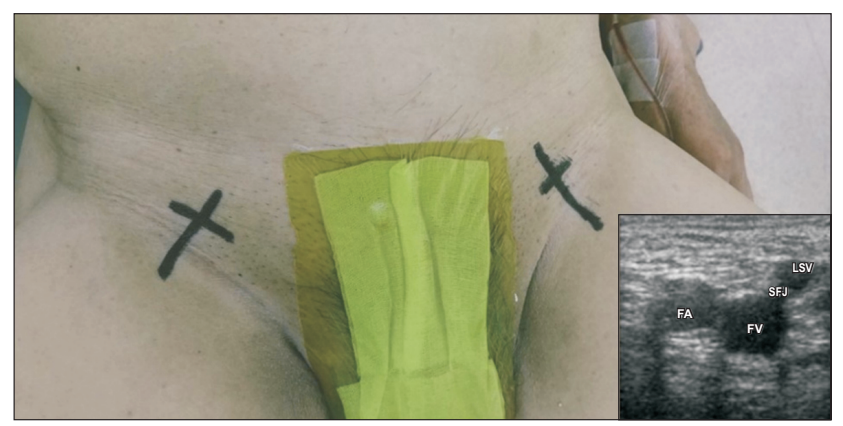

Fig. 1. At the beginning of surgery, the path and bifurcation of the femoral artery (FA) were checked via sonographic guidance, and pre-marking on the skin was performed. The requirement for individual consent was waived. FV, femoral vein; SFJ, sapheno-femoral junction; LSV, long saphenous vein. 
nulation. The "pre-closure" was applied to the FA with 2 ProGlide devices [8]. The first device was inserted into the FA over the guide wire at a $45^{\circ}$ angle, and the pre-closing suture was deployed with rotation at 10 o'clock relative to the patient's head. The second device was inserted over the guidewire, and the suture was deployed using the same maneuver, except with rotation at 2 o'clock [8]. After the pre-closure, the arterial guide wire was introduced under TEE guidance in the descending thoracic aorta. Finally, the arterial cannula was inserted via the pre-closure applied to the FA and then connected to the CPB.

For decannulation, after stopping the $\mathrm{CPB}$ machine, the venous cannula was removed and controlled first with a figure-of-eight skin suture and manual compression. The arterial cannula was removed after protamine infusion. Two preformed knots were tightened with a suture trimmer enclosed with ProGlide. Additional manual compression was applied for several minutes (usually 5-10 minutes) by assistants, if needed, and the skin was closed with a simple interrupted suture (Supplementary Video 1).

\section{Definition of procedure success and assessment of postoperative arterial stenosis}

Procedural success was defined as successful hemostasis of the FA without additional surgical or interventional procedures. Cannulation-related complications were defined as events that occurred at the arterial cannulation site, including bleeding, FA stenosis, lymphocele, infection, pseudo-aneurysm, and paresthesia.

The dorsal pulse was checked immediately after surgery, and the arterial pulse was assessed with pulse oximetry on the toe of the cannulation side during management in the intensive care unit. To assess FA stenosis, the ankle-brachial index (ABI) was measured postoperatively.

\section{Statistical analysis}

Quantitative variables are reported as the mean \pm standard deviation. Discrete variables are presented as numbers and percentages. The Mann-Whitney U-test was used to compare quantitative variables, and the chi-square test or Fisher exact test was performed for discrete variables. The independent t-test was used to compare the results of ABI. For all statistics, 2-sided p-values were used, and statistical significance was defined as a $p$-value $<0.05$. Statistical analyses were performed with $\mathrm{R}$ software ver. 3.4.0 (The R Foundation for Statistical Computing, Vienna, Austria; http://www.r-project.org/).

\section{Results}

\section{Baseline characteristics and operative data}

The clinical baseline characteristics are presented in Table 1 . The mean age of subjects was $55.7 \pm 15.5$ years, and the sex distribution was relatively even. The mean value of the body surface area was $1.7 \mathrm{~m}^{2}$. Patients' body weight ranged from 40.5 to $87.6 \mathrm{~kg}$, and their height ranged from 142.5 to $186.9 \mathrm{~cm}$. More than one-fifth of patients underwent an urgent or emergent operation. More than $95 \%$ of patients had an FA $>6 \mathrm{~mm}$ on preoperative CTA. For most patients, $15 \mathrm{~F}$ to $19 \mathrm{~F}$ cannulas were used for arterial cannulation. The distribution of the size of the cannula according to the size of the femoral artery was summarized in Supplementary Table 1 . The right-side FA was mostly used for cannulation $(98.3 \%)$ because the cannulation was mainly performed on the surgeon's side. However, left-side cannulation was performed when the left FA was considered more suitable for anatomical reasons, such as size, tortuosity, or less calcification. Most of the procedures were MICS, and the others needed femoral cannulation, such as during redo cardiac surgery. Most of the subjects were patients who underwent MICS, but some underwent sternotomy $(n=6)$. FA cannulation in sternotomy was used in redo sternotomy patients with severe adhesion between the great vessels and sternum, or in patients in whom central cannulation was difficult. The surgical details are also described in Table 2.

\section{Clinical outcomes and procedure-related complications during hospitalization}

Three cases of early mortality were recorded. The causes of death were multiorgan failure in 1 patient who underwent heart transplantation, sepsis in 1 patient who received tricuspid valve replacement due to infective endocarditis, and low cardiac output syndrome in 1 patient who underwent biatrial ablation of atrial fibrillation, tricuspid valve repair, and atrial septal defect (ASD) closure. However, in these 3 patients, no complications related to FA cannulation were recorded.

More than $60 \%$ of patients underwent immediate extubation of the endotracheal tube in the operating room. Despite early movements of the extremities in these patients, no complications related to incomplete hemostasis or delayed bleeding of the cannulation site were noted. Fewer than one-third of patients received transfusions with packed red blood cells during or after surgery. The rates of 
other major complications were comparable (Table 3).

All FA cannulations were successfully controlled with ProGlide, except in 1 patient who needed a small skin incision to complete hemostasis. There was only 1 case of ProGlide failure, which was believed to be due to the lack of technical proficiency during our early experience. In this case, despite more than 30 minutes of manual compression after decannulation with applying ProGlide, active bleeding from the arterial cannulation site continued. To determine the cause of hemostasis failure, a $5-\mathrm{cm}$ incision was

Table 2. Operative profiles $(n=118)$

\begin{tabular}{|c|c|}
\hline Variable & Value \\
\hline Urgent or emergent operation & $24(20.3)$ \\
\hline Mitral valve & $9(7.6)$ \\
\hline Aortic valve & $5(4.2)$ \\
\hline Tricuspid valve & $2(1.7)$ \\
\hline Combined operation & $4(3.4)$ \\
\hline Others (ASD, thrombus removal, etc.) & $4(3.4)$ \\
\hline Infective endocarditis & $10(8.5)$ \\
\hline Minimally invasive cardiac surgery & $112(94.9)$ \\
\hline Mitral valve & $28(23.7)$ \\
\hline Aortic valve & $27(22.9)$ \\
\hline Tricuspid valve & $7(5.9)$ \\
\hline Combined operation & $16(13.6)$ \\
\hline Others (ASD, myxoma, etc.) & $34(28.8)$ \\
\hline Sternotomy & $6(5.1)$ \\
\hline Redo operation & $4(3.4)$ \\
\hline Aorta surgery & $1(0.8)$ \\
\hline Heart transplantation & $1(0.8)$ \\
\hline Cardiopulmonary bypass time (min) & $108.6 \pm 62.8$ \\
\hline Immediate extubation in the operation room & $72(62.6)$ \\
\hline \multicolumn{2}{|l|}{ Cannulation side } \\
\hline Right femoral artery & $116(98.3)$ \\
\hline Left femoral artery & $2(1.7)$ \\
\hline \multicolumn{2}{|l|}{ Size of arterial cannula } \\
\hline $14 \mathrm{~F}$ & $1(0.8)$ \\
\hline $15 \mathrm{~F}$ & $58(49.2)$ \\
\hline $16 \mathrm{~F}$ & $1(0.8)$ \\
\hline $17 \mathrm{~F}$ & $39(33.1)$ \\
\hline $19 \mathrm{~F}$ & $19(16.1)$ \\
\hline
\end{tabular}

Values are presented as number (\%) or mean \pm standard deviation. ASD, atrial septum defect. made, and the FA was exposed. The preformed knot of ProGlide was located in the subcutaneous tissue, not the FA, and active bleeding of the FA persisted. The bleeding site of the FA was repaired with a double layer of 5-0 Prolene, and the surgical wound was repaired. It is presumed that the device was pulled back when anchoring the preclosing suture. Otherwise, no cases required surgical repair or additional procedures for hemostasis during hospitalization. Moreover, there was no delayed bleeding, lymphocele, pseudo-aneurysm, wound problem, or paresthesia in any patient during hospitalization.

\section{Assessment of postoperative FA stenosis}

Postoperative ABI tests were routinely performed after the initial 26 cases to assess functional stenosis of the FA $(n=81,68.6 \%)$. The postoperative ABI tests showed no functional stenosis, except in 1 patient, when the cannulation side was compared with the non-cannulation side. The postoperative ABI values of both sides were within the normal range of the $\mathrm{ABI}$, which is from 1.1 to 1.3 (cannulation side [1.16 \pm 0.12$]$ versus non-cannulation side [1.12 \pm 0.10$]$, $\mathrm{p}<0.001)$. In addition, 27 patients underwent preoperative $\mathrm{ABI}$ testing, and no difference was found between the preoperative and postoperative ABI values of the cannulation side $(1.15 \pm 0.13$ versus $1.18 \pm 0.11, p=0.158)$. The details of the ABI test are described in Table 4 . The subgroup analy-

Table 3. Adverse clinical outcomes $(n=118)$

\begin{tabular}{lc}
\hline \multicolumn{1}{c}{ Variable } & No. $(\%)$ \\
\hline During hospitalization & $3(2.6)$ \\
$\quad$ Early mortality (within 30 days) & $1(0.8)$ \\
Cerebrovascular accident & $2(1.7)$ \\
Acute renal failure & $33(30.0)$ \\
Transfusion of red blood cells & \\
Complications at the cannulation site & $1(0.8)$ \\
$\quad$ Device failure (open repair) & \\
Device-related complications during follow-up & $1(0.8)^{\mathrm{a})}$ \\
$\quad \begin{array}{l}\text { Claudication of the leg } \\
\text { Interventional procedure }\end{array}$ & $1(0.8)^{\mathrm{a})}$ \\
\hline
\end{tabular}

a) Event in the same patient.

Table 4. Results of postoperative ankle brachial pulse index $(n=81)$

\begin{tabular}{lccc}
\hline \multicolumn{1}{c}{ Variable } & Cannulation side & Non-cannulation side & p-value \\
\hline Overall result & $1.2 \pm 0.1$ & $1.1 \pm 0.1$ & $<0.001$ \\
According to femoral artery size & & & \\
Smaller than $7 \mathrm{~mm}(\mathrm{n}=12)$ & $1.2 \pm 0.1$ & $1.1 \pm 0.1$ & 0.38 \\
$7<$ size $\leq 10 \mathrm{~mm}(\mathrm{n}=38)$ & $1.2 \pm 0.1$ & $1.1 \pm 0.1$ & 0.035 \\
Larger than $10 \mathrm{~mm}(\mathrm{n}=31)$ & $1.2 \pm 0.1$ & $1.1 \pm 0.1$ & 0.22 \\
\hline
\end{tabular}

Values are presented as mean \pm standard deviation. 
sis of patients stratified by the size of FA showed no significant difference in the postoperative ABI value between the cannulation side and the non-cannulation side.

\section{Procedure-related complications during follow-up}

One female patient, who underwent MICS ASD patch closure, presented with pain and numbness of the right lower leg during a visit to the outpatient clinic. The postoperative ABI of the right lower leg had decreased to 0.73 from 0.98. CTA showed stenosis of the right common FA, and the patient was treated by percutaneous balloon angioplasty (Fig. 2A, B). After the intervention, the pain and numbness improved, and the follow-up ABI of the right lower leg increased to 0.93 , which was within the normal range. No other complications associated with femoral cannulation, such as wound problems, hematoma, and lymphocele, were recorded during follow-up.

\section{Discussion}

This study evaluated 118 patients who underwent cardiac surgery using ProGlide for percutaneous FA cannulation and showed a good success rate and a low complication rate. ProGlide was applied in more than $90 \%$ of patients who required FA cannulation, and the procedure success rate with this method was $99.2 \%$. During follow-up, only 1 patient was treated with balloon dilation for stenosis of the FA, and no other complications were reported in other patients. Moreover, the use of ProGlide with percutaneous FA cannulation during cardiac surgery was feasible and safe.

Previous studies have proved the safety and feasibility of the PVCD during interventional procedures [3-6]. In particular, ProGlide was found to perform better than other PVCDs in interventional research [9-13]. However, in cardiac surgery, only a few studies have reported results from
PVCD use. Ramponi et al. [7] introduced total percutaneous CPB using ProGlide in 2011, and Moschovas et al. [8] reported their experience using ProGlide in 353 patients who underwent MICS using femoral cannulation. They revealed that percutaneous cannulation using ProGlide significantly reduced groin complications, operation time, and hospital stay. Excluding these, however, no report, to the best of our knowledge, have focused on the outcomes of PVCDs with cardiac surgery.

Nevertheless, the frequency and demand for the use of PVCD in cardiac surgery are increasing gradually. With the start of the MICS era, the frequency of using femoral cannulation has increased [1]. In addition, as the life span of patients lengthens and cardiac surgery becomes more common, the number of patients undergoing repeated cardiac surgery, such as heart transplantation or ventricular-assisted device procedures, is increasing. In such cases, femoral cannulation can be an important option for reducing the difficulty of surgery and ensuring safety. The traditional approach of femoral cannulation is to make a small incision in the inguinal area and to access the femoral vessels (Fig. 3A, B) [2,6]. The open surgical technique for femoral cannulation may have some disadvantages compared
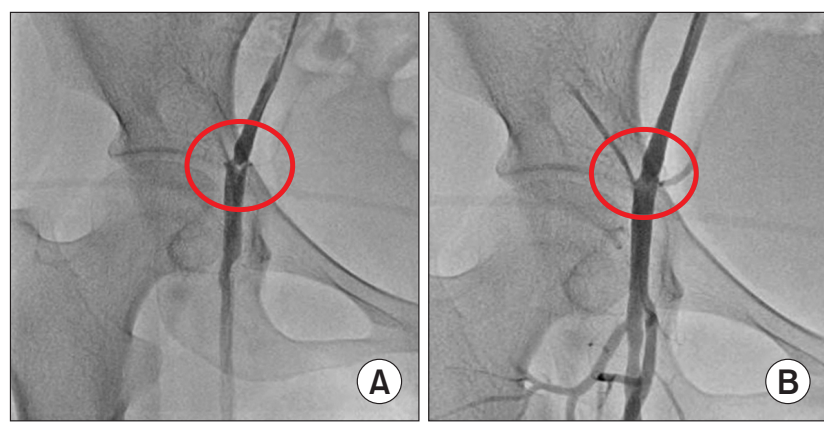

Fig. 2. Stenosis of the right femoral artery (A) was treated via balloon angiography (B) (circles).
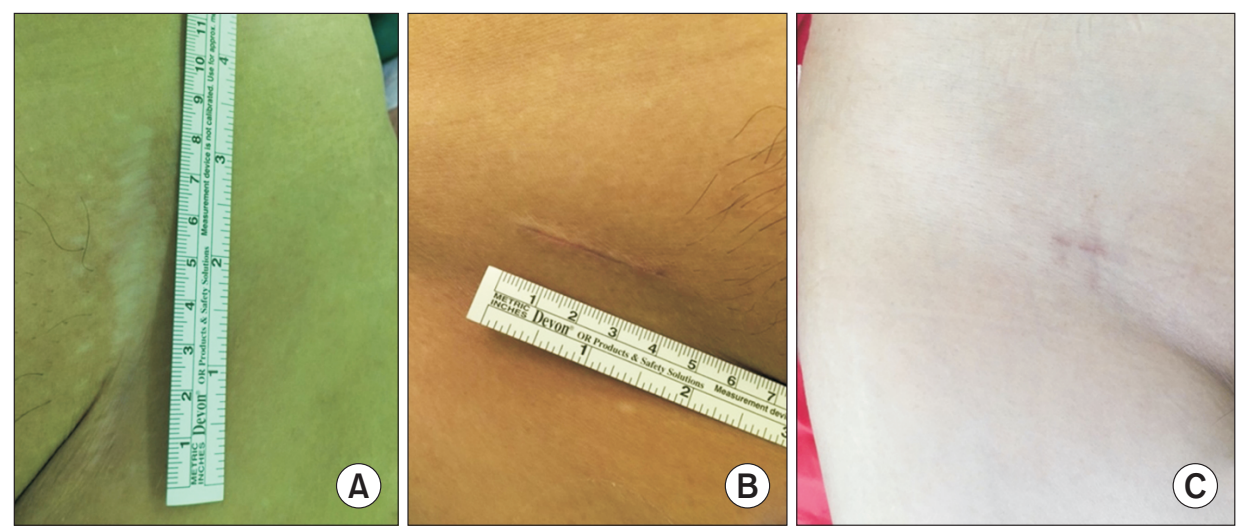

Fig. 3. The incision traditionally performed for accessing the femoral artery was $>3 \mathrm{~cm}$ in length $(\mathrm{A}, \mathrm{B})$ and has various complications in the postoperative period. (C) The incision for femoral cannulation using the ProGlide (Abbott Vascular Inc., Santa Clara, CA, USA) is much smaller than the traditional incision and leads to fewer complications. The requirement for individual consent was waived. 
to percutaneous cannulation. Due to the incision and dissection of subcutaneous tissue, there is an increased possibility of surgical wound complications such as infection, lymphocele, and hematoma. Additionally, the open surgical technique may increase pain in the inguinal area, which may result in delayed ambulation and reduced patient satisfaction. In this case, the characteristics of the surgical wound or the occurrence of complications, such as lymphocele and paresthesia, may decrease patients' satisfaction with the surgery and increase the length of stay or frequency of hospital visits. From this point of view, percutaneous cannulation using PVCD can increase patient satisfaction related to the surgical wound and prevent complications, such as lymphocele and paresthesia $[4,6,8]$. In this study, no patient reported any complications related to the femoral wound (Fig. 3C).

The use of PVCD in cardiac surgery has some difficulties, and the indications and instructions for its use are not well established. Compared with interventional procedures, cardiac surgery requires higher concentrations of heparin and maintenance of cannulation for a longer time. In addition, the required extracorporeal circulation and hypothermia interfere with hemostasis after cardiac surgery. Realistically, the routine use of fluoroscopy is difficult during cardiac surgery. Under these conditions, accurate puncture of the common FA is important to increase the success rate of the device and to prevent postoperative stenosis or occlusive complications [11-13]. In this study, we were able to perform percutaneous cannulation in more than $90 \%$ of patients who required femoral cannulation through risk stratification using preoperative CTA. In addition, the pre-marking technique showed a success rate of $>99 \%$ in terms of its application and hemostasis with FA cannulation using ProGlide.

Postoperative stenosis or occlusive complications are a concern related to PVCD, especially in patients with a small FA [11,13]. Enhanced CTA or vascular angiography is needed to identify these anatomical complications. However, these tests are not appropriate for screening because of their invasiveness and high cost. The ABI is suitable for screening because it is simple, non-invasive, and inexpensive. Most previous studies did not rigorously exclude the possibility of postoperative stenosis based on an objective examination. To our knowledge, the present study is the first to describe the results of postoperative evaluations of FA stenosis by a routine screening test with ABI after the use of PVCD during cardiac surgery. According to our results, the ABI value of the cannulation side did not decrease compared with that of the non-cannulation side. In particular, no significant reduction in ABI was noted in the subgroup analysis of patients with an FA $<7 \mathrm{~mm}$ (Table 4).

Moreover, concerns have been raised that the use of ProGlide in small FAs may be more likely to cause stenosis. Based on our experience, some tips can be derived for applying ProGlide in small FAs. First, preoperative CTA should be carefully reviewed and the surgeon should set his or her own criteria for the size of the FA for using ProGlide. Second, the pre-marking method is particularly important in patients with small FAs for puncturing the common FA just above the bifurcation. Third, it is important to open the foot of the ProGlide device and deploy the suture at the starting point where the pulsation of blood is regurgitated through the marker lumen of the ProGlide. Deployment of the suture with the ProGlide in the deep position can capture the posterior wall of the vessel and induce FA stenosis postoperatively (Fig. 4).

In conclusion, the use of ProGlide was safe and feasible in adult patients who needed percutaneous FA cannulation during cardiac surgery. This method showed effective hemostasis and reduced cannulation-related complications. We believe that the present technique may be a good alter-

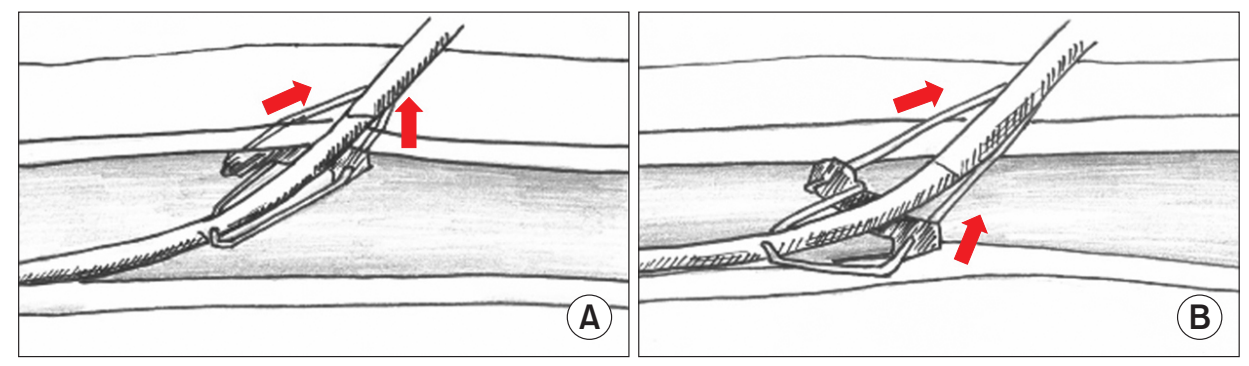

Fig. 4. When the foot of the ProGlide (Abbott Vascular Inc., Santa Clara, CA, USA) is opened at the starting point of pulsating blood through the marker lumen of the ProGlide (A), the suture of the ProGlide may be applied on the anterior wall of the femoral artery (arrows). Opening the foot of the ProGlide and deploying the suture in the deep position can capture the posterior wall of the vessel (B, arrows). 
native to the conventional method in patients who need FA cannulation during cardiac surgery.

This study has some limitations. First, this observational study has some potential limitations due to its retrospective design. Second, this study has a relatively small sample size from a single institution. Finally, there was no appropriate control group within the study period. However, the methodology was consistent, as the study was carried out at a single institution and highly reliable data were collected. The homogeneity of operative strategies of consecutive cases from a single center strengthens the credibility of the study findings. We hope that further extensive, prospective, randomized studies will be performed based on the results presented herein.

\section{Conflict of interest}

No potential conflict of interest relevant to this article was reported.

\section{ORCID}

Chang Hun Kim: https://orcid.org/0000-0003-3181-7454

Min Ho Ju: https://orcid.org/0000-0001-7839-8598

Mi Hee Lim: https://orcid.org/0000-0002-0167-7836

Chee-Hoon Lee: https://orcid.org/0000-0002-0456-225X

Hyung Gon Je: https://orcid.org/0000-0003-4713-2898

\section{Supplementary materials}

Supplementary materials can be found via https://doi. org/10.5090/jcs.21.006. Supplementary Table 1. Distribution of cannula sizes according to the size of the femoral artery. Supplementary Video 1. The use of ProGlide in femoral cannulation during cardiac surgery.

\section{References}

1. Chan EY, Lumbao DM, Iribarne A, et al. Evolution of cannulation techniques for minimally invasive cardiac surgery: a 10-year journey. Innovations (Phila) 2012;7:9-14.

2. Cakici M, Yazicioglu L, Baran $\mathrm{C}$, et al. A retrospective analysis of surgical femoral artery closure techniques: conventional versus purse suture technique. Ann Vasc Surg 2017;44:103-12.

3. Robertson L, Andras A, Colgan F, Jackson R. Vascular closure devices for femoral arterial puncture site haemostasis. Cochrane Database Syst Rev 2016;3:CD009541.

4. Karaolanis G, Kostakis ID, Moris D, Palla VV, Moulakakis KG. Fascia suture technique and suture-mediated closure devices: systematic review. Int J Angiol 2018;27:13-22.

5. Kodama A, Yamamoto M, Shimura T, et al. Comparative data of single versus double ProGlide vascular preclose technique after percutaneous transfemoral transcatheter aortic valve implantation from the optimized catheter valvular intervention (OCEAN-TAVI) Japanese multicenter registry. Catheter Cardiovasc Interv 2017;90:E55-62.

6. Steppich B, Stegmuller F, Rumpf PM, et al. Vascular complications after percutaneous mitral valve repair and venous access closure using suture or closure device. J Interv Cardiol 2018;31:223-9.

7. Ramponi F, Yan TD, Vallely MP, Wilson MK. Total percutaneous cardiopulmonary bypass with Perclose ProGlide. Interact Cardiovasc Thorac Surg 2011;13:86-8.

8. Moschovas A, Amorim PA, Nold M, et al. Percutaneous cannulation for cardiopulmonary bypass in minimally invasive surgery is associated with reduced groin complications. Interact Cardiovasc Thorac Surg 2017;25:377-83.

9. Dall'Ara G, Santarelli A, Marzocchi A, et al. Vascular complications after balloon aortic valvuloplasty in recent years: incidence and comparison of two hemostatic devices. Catheter Cardiovasc Interv 2018; 91:E49-55

10. Barbash IM, Barbanti M, Webb J, et al. Comparison of vascular closure devices for access site closure after transfemoral aortic valve implantation. Eur Heart J 2015;36:3370-9.

11. Nara Y, Watanabe Y, Kozuma K, et al. Incidence, predictors, and mid-term outcomes of percutaneous closure failure after transfemoral aortic valve implantation using an expandable sheath (from the Optimized Transcatheter Valvular Intervention [OCEAN-TAVI] registry). Am J Cardiol 2017;119:611-7.

12. Zakko J, Scali S, Beck AW, et al. Percutaneous thoracic endovascular aortic repair is not contraindicated in obese patients. J Vasc Surg 2014;60:921-8.

13. Hu G, Chen B, Fu W, et al. Predictors and treatments of ProGlide-related complications in percutaneous endovascular aortic repair. PLoS One 2015;10:e123739. 